\title{
ULTRASONICALLY INDUCED INTRAVASCULAR STREAMING AND THROMBUS FORMATION ADJACENT TO A MICROPIPETTE
}

\author{
L. A. Frizzell, ${ }^{*}$ D. L. Miller and W. L. NyBorG \\ Department of Physics, Cook Physical Sciences Building, University of Vermont, Burlington, VT 05405, U.S.A.
}

(Received 13 June 1985; in final form 31 October 1985)

\begin{abstract}
Ultrasonically induced microstreaming around bubbles of gas have been shown to cause damage to biological materials in vitro at clinical exposure levels. The potential for ultrasonically induced cavitation and microstreaming in mammalian systems is of interest with respect to the safe application of clinical ultrasound. Ultrasonically induced intravascular microstreaming and formation of thrombi at $1 \mathrm{MHz}$ is demonstrated adjacent the tip of a micropipette in contact with a mesenteric vessel of the mouse. These effects occur at or above therapeutic levels.
\end{abstract}

Key Words: Ultrasonics, Biological effects, Micropipette, Mouse, Streaming, Thrombus.

\section{INTRODUCTION}

Several reports have been published recently which summarize the current understanding of the biological effects of ultrasound (Stewart and Stratmeyer, 1982; NCRP Report, 1983; WHO Report, 1982). It is apparent from these summaries that several mammalian studies demonstrating biological effects associated with cavitation have shown these effects to occur at very high intensities (Fry et al., 1970; Dunn and Fry, 1971; Chan and Frizzell, 1977; Frizzell et al., 1983). In another in vivo study (Gross et al., 1985) no bubble formation was observed in cardiac blood due to ultrasound exposure at spatial peak intensities up to $16 \mathrm{~W} /$ $\mathrm{cm}^{2}$. However, cavitation produces biological effects at low intensities in solutions and suspensions, in plants, and in insects. Bubbles can be formed in mammalian systems by ultrasound at therapeutic levels (ter Haar and Daniels, 1981; ter Haar et al., 1982). In addition, Lehmann and Herrick (1953) have reported the formation of petechial hemorrhages in mice in vivo at low ultrasound exposures. Dependence upon pressure, temperature, and frequency was consistent with cavitation. Therefore, the potential role of cavitation in vivo needs further investigation.

One critical question concerns the effect of cavitation-induced microstreaming on blood. In vitro cavitation can cause platelet aggregates to form near-stable

* Permanent address: Bioacoustics Research Laboratory, University of Illinois, 1406 West Green Street, Urbana, IL 61801, U.S.A. microbubbles in platelet-rich plasma (Miller et al., 1979). Williams (1977) used a $20 \mathrm{kHz}$ vibrating wire, which simulates the action of a cavitation bubble, to demonstrate in vivo thrombus formation by acoustic microstreaming. This result has apparently not been obtained previously in vivo at medically relevant frequencies. Herein we report the occurrence of microstreaming and thrombus formation within blood vessels in contact with a micropipette exposed to $1 \mathrm{MHz}$ ultrasound.

\section{MATERIALS AND METHODS}

ICR Swiss mice (Charles River) were anesthetized with $0.39 \mathrm{M}$ chloro hydrate administered intraperitoneally at a dose of $0.01 \mathrm{ml} / \mathrm{gm}$ body weight. The abdomen was opened via a midline incision, and the gut was exteriorized. The intestines were spread and held in place by sutures on a microscope stage such that the mesenteric arteries and veins were accessible from above and placed over the aperture in the stage. The stage of the microscope was modified to include a quartz source with a small aperture in the center of the electrodes to pass light from below the stage. Thus, any transparent specimen placed on the quartz ultrasound source could be sonicated and viewed through the microscope simultaneously. A more complete description of the microscope and stage can be found elsewhere (Miller, 1976).

Micropipettes (Frederick Haer \& Co.) approximately $2 \mu \mathrm{m}$ in diameter at the tip were placed such 
that the tip or the tapered section immediately behind the tip was laying on top of one of the mesenteric vessels. This preparation was then covered with a laycr of physiological saline to provide coupling between the source and tissue and to immerse the micropipette. The voltage applied to the source was adjusted to the desired level and the blood flow in the vessel was observed to detect any streaming resulting from the ultrasound. In order to detect unambiguously very low velocity streaming, the ultrasound was usually pulsed one second on and one second off with an audio tone indicating when the sound was on.

Several sections of vessels were removed and processed for examination with the light microscope by either removing the section and fixing or by partially fixing and then removing. All samples were fixed with $10 \%$ neutral buffered formalin, dehydrated through graded alcohol, embedded in paraplast, and cut at 5 $\mu \mathrm{m}$. Cut and mounted sections were stained with either hematoxylin and eosin or Masson's trichrome.

The ultrasonic field was not measured in this study because no method currently available is capable of readily providing the required data. The $0.987 \mathrm{MHz}$ field employed is a standing wave with pressure and velocity amplitudes which probably vary considerably from specimen to specimen, and from point to point in the field. Since the phenomenon observed apparently depends on acoustic streaming generated at the tip of a micropipette, the relevant field parameter is the velocity amplitude at the tip. This parameter was maximized by placing the micropipette so that it was near the surface of the bathing liquid. This water-air interface presents a free surface boundary condition to the field, which results in the creation of a velocity amplitude maximum at the interface regardless of the shape of the surface.

Although the field was not measured, it is possible to estimate the velocity amplitudes produced by a given input voltage from previous measurements of the fields generated by this instrument under more easily defined conditions (Miller, 1976). At an input voltage of 50 $\mathrm{V}_{\mathrm{rms}}$, the displacement of the surface of the quartz transducer was found to be about $15 \mathrm{~nm}$, with a droplet of water in place. This displacement amplitude corresponds to a radiated (plane wave) velocity amplitude of about $10 \mathrm{~cm} / \mathrm{s}$. Within a precisely formed cylindrical droplet (which behaves like a resonant cavity), pressure amplitudes were found to range up to 5 bar (0.5 MPa) which corresponds to a velocity amplitude of about 33 $\mathrm{cm} / \mathrm{s}$. It seems plausible to specify the range $10-33 \mathrm{~cm} /$ $\mathrm{s}$ at $50 \mathrm{~V}_{\mathrm{rms}}$ input as an estimate for the velocity amplitude in this study. For a plane wave, this velocity amplitude range would correspond to an intensity range of about $0.75-8.3 \mathrm{~W} / \mathrm{cm}^{2}$.

\section{RESULTS}

The mesenteric vessels of 12 adult mice werc exposed to $1 \mathrm{MHz}$ ultrasound while in contact with a micropipette, and the blood flow within the vessels was observed through the microscope. Acoustic streaming of the blood within the vessels was often visible at applied voltages of less than $10 \mathrm{~V}_{\text {rms }}$ and was virtually always visible at $50-100 \mathrm{~V}_{\mathrm{rms}}$. Figure 1 shows a series of three photomicrographs in temporal sequence for Mouse \#8 with the sound on, off, and on, respectively. These particular photomicrographs were taken immediately after death, but are representative of the streaming which occurs in the live mouse. The flow lines and the clear areas of separation between regions of rapidly streaming blood (vortices) and regions where little or no acoustic streaming occurs are clearly visible in Fig. 1(a) and 1(c).

Detection of the acoustic streaming depended upon the acoustic streaming velocity induced in a particular configuration and the normal blood flow velocity. The streaming was more readily observed in veins than in arteries. As might be expected, the greater the blood flow the greater the streaming velocity required for observation of an effect. This threshold of observability may have been related to the level at which the tendency for acoustic streaming flow could overcome the blood flow and stop or reverse the flow in parts of the vessel.

The streaming velocity at a given applied voltage was dependent upon location in the field and required submersion of the vessel and micropipette tip. On several occasions vigorous streaming would be observed for a while, but would later disappear. It was soon discovered that the streaming would return after addition of more coupling medium which had evaporated with time. Also, it was found that similar streaming occurred when the tip of the micropipette penetrated the vessel wall.

In many cases where very vigorous streaming was observed clumps (thrombi) were formed adjacent to the tip of the pipette, which modified the blood flow even with the ultrasound off. Figure 2 shows one of these thrombi as it appeared under the experimental conditions. These thrombi almost always remained, attached to the vessel wall, when the sound field and micropipette were removed. Several sections of vessel containing thrombi were removed, fixed, and processed for examination with the light microscope. Figure 3 shows a thrombus after preparation with Hematoxylin and Eosin staining. The thrombus is clearly visible and is attached to, or involves, the vessel wall. Thrombi formed whenever vigorous streaming occurred. They would of course disrupt the pattern of blood flow, and 


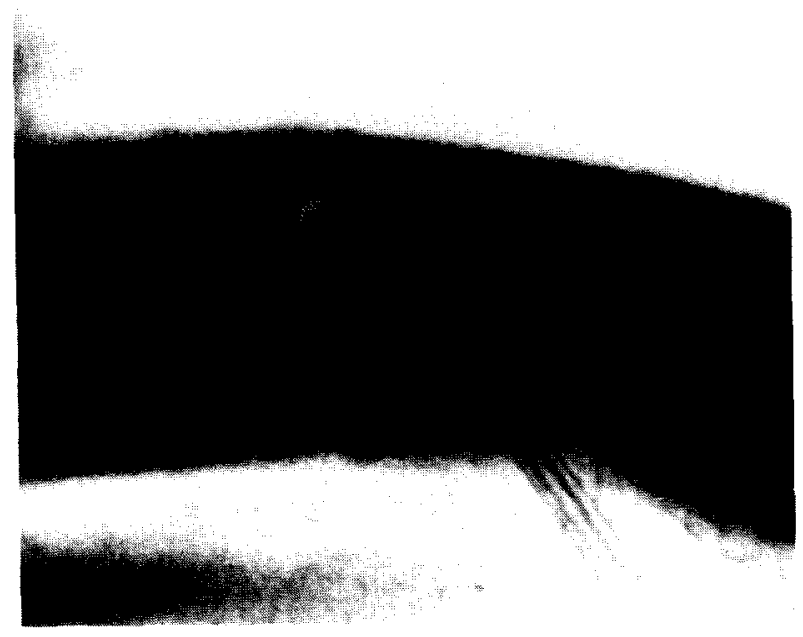

(a)

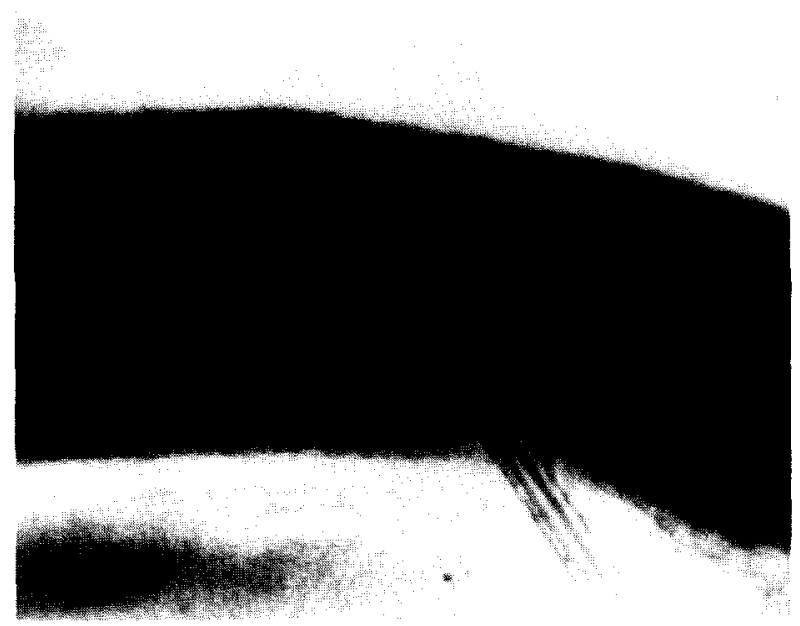

(b)

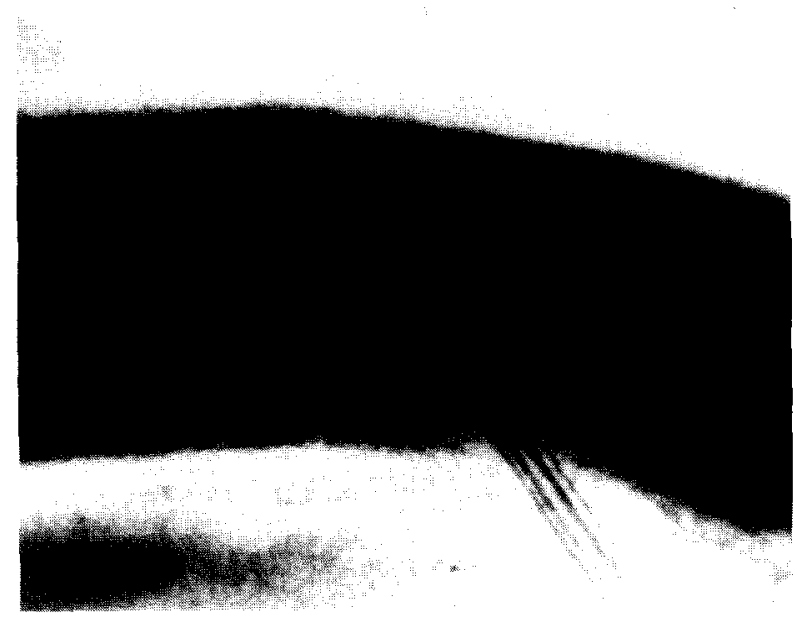

(c)

Fig. 1. Photomicrographs of a micropipette placed on a mesenteric vein of Mouse \#8, in sequence: (a) sound on; (b) sound off; and (c) sound on. Streamlines and vortices are apparent in (a) and (c), but absent in (b). Applied voltage was $48 \mathrm{~V}_{\text {rms }}$.

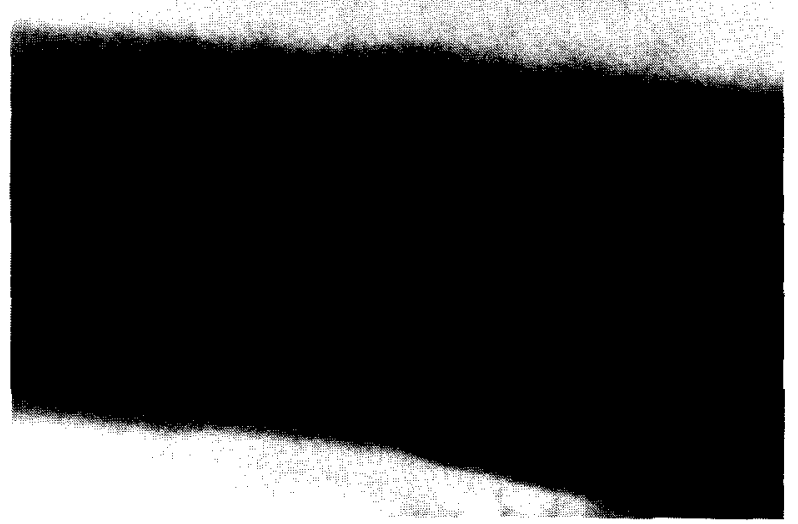

Fig. 2. Photomicrograph of a thrombus adjacent the micropipette tip in Mouse \#9, sound off. Thrombus was created with an applied voltage of $50 \mathrm{~V}_{\mathrm{rms}}$.

their formation decreased the apparent acoustic streaming velocity, presumably because the thrombus formed immediately adjacent to the tip of the micro-

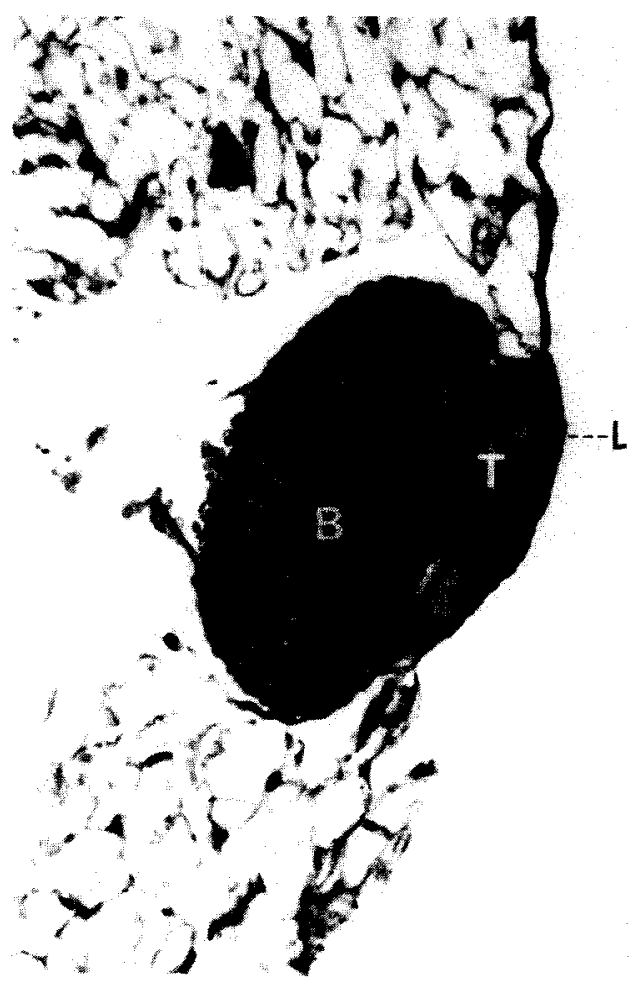

Fig. 3. Photomicrograph of a microscope section (H \& $E$ stain) containing the cross section of a vessel in Mouse \#15 clearly showing the residual blood (B), which occurs because the animal is not perfused, and the thrombus ( $T$ ) attached to the surface that the micropipette had contacted. Applied voltage was $100 \mathrm{~V}_{\mathrm{rms}}$. The thick dark layer (L) on the right outside surface of the vessel and surrounding tissues is the India ink that was used to mark the position of the thrombus prior to removal. 
pipette. On at least one occasion a thrombus appeared to form but break away from the pipette and disappear downstream. However, almost all thrombi that were observed adhered to the vessel wall.

\section{DISCUSSION}

The perturbations in blood flow, clot formation and possible vessel-wall damage observed in this study are all presumably directly related to the acoustic streaming generated at the tip of the micropipette. Clot formation seemed to occur at roughly $50 \mathrm{~V}_{\text {rms }}$ input, which according to the estimate from the Methods section produced velocity amplitudes of roughly 10-33 $\mathrm{cm} / \mathrm{s}$. Although there are uncertainties in the field specification and in the precise nature of the coupling of the micropipette tip to the vessel wall, it is possible to make an order-of-magnitude calculation of the acoustic streaming expected at the tip. Assuming that the tip remains fixed and that fluid oscillates around it, a boundary layer is set up at the stationary surface (possibly the inside surface of the blood vessel) of thickness $l$ given by

$$
l=(\mu / \pi \rho f)^{1 / 2},
$$

in which $\mu$ is the viscosity of the fluid, $\rho$ is its density and $f$ is the oscillation frequency. Within this boundary layer, steady forces are set up which lead to fluid flow (Nyborg, 1965). The limiting flow, or streaming velocity $U$ just outside this layer is given by

$$
U=u^{2} / 2 \pi a f
$$

in which $u$ is the oscillatory velocity amplitude, and $a$ is the radius of the cylindrical micropipette tip. The acoustic streaming generates a velocity gradient which may be approximated by $U / l$, and a shear stress given by

$$
S=\mu U / l .
$$

It should be noted that although this is a somewhat simplified theory, it has proven to be quite useful for assessing the magnitude of the shear stress mechanism of ultrasonic bioeffects (see, for example, Williams $e t$ al., 1970).

In this study, the frequency was about $1 \mathrm{MHz}$, and the fluid viscosity might be estimated at $2 \mathrm{cP}$ (appropriate for blood plasma). The boundary layer thickness is thus $l=0.8 \mu \mathrm{m}$. For velocity amplitudes $u$ of $10-33 \mathrm{~cm} / \mathrm{s}$, streaming velocities $U$ are expected to be in the range of $0.15-1.5 \mathrm{~cm} / \mathrm{s}$ for a micropipette radius $a=1 \mu \mathrm{m}$ (it should be noted that the unknown radius of curvature at the open end of the micropipette may be smaller than $1 \mu \mathrm{m}$, so that this may be a conservative estimate). Resulting shear stresses $S$ range from about $40-400 \mathrm{dyn} / \mathrm{cm}^{2}$. Shear stresses of this magnitude are sufficient to damage blood platelets (Brown et al., 1975).

Conditions in this study seem to be analogous to those in the study by Williams (1977), except that Williams employed a $115 \mu \mathrm{m}$ radius wire oscillating transversely at $20 \mathrm{kHz}$. It is interesting to compare these two situations on the basis of the acoustic streaming theory. Williams observed clot formation in the femoral vein of the mouse for a displacement amplitude of about $10 \mu \mathrm{m}$. This displacement amplitude corresponds to a velocity amplitude relative to the fluid of about $125 \mathrm{~cm} / \mathrm{s}$. The acoustic streaming velocity calculated from eqn 2 is about $11 \mathrm{~cm} / \mathrm{s}$, with a boundary layer thickness of $5.6 \mu \mathrm{m}$. The shear stress expected in Williams' situation when clot formation occurred was therefore about $400 \mathrm{dyn} / \mathrm{cm}^{2}$. Note that this estimate is the same as the estimated upper limit for this study at $1 \mathrm{MHz}$. A plausible conclusion is that this and Williams' study are of the same biophysical phenomenon occurring at two widely different frequencies. One case involves a wire oscillating in a fixed fluid and the other case involves a fixed micropipette in an oscillating fluid.

It is also of interest to compare the results of this study to those of Martin et al. (1983) who observed microstreaming within the blood vessels in the tail of the Yellow Comet Platy Fish. The tails were mounted on an air-backed window and sonicated with $0.78,1.5$, and $3.0 \mathrm{MHz}$ ultrasound. Blood flow was observed through the window using a light microscope. Microstreaming associated with the ultrasound at peak intensities of a few W/ $\mathrm{cm}^{2}$ was observed first in positions where capillaries crossed the edges of cartilaginous rods. They also observed microbubbles within the fish that were believed to originate from the water in the tank and noted that microstreaming occurred around some of these. However, no microbubbles were associated with the streaming near the rods, and this streaming was not correlated with subharmonic detection as an indicator of cavitation. Thus results reported here would seem to confirm that the microstreaming observed by Martin and coworkers was associated with the rigid boundary of the rods. Additionally, the intensity levels are comparable for the two studies.

The significance of these results are not yet clear. The finding that ultrasound in the clinical frequency range can cause microstreaming and formation of thrombi adjacent to a small-diameter rigid object is of much interest. Our rough estimates of the irradiating field would indicate that the intensities necessary to produce streaming are at therapeutic levels or higher. These initial results suggest the need for a more-detailed 
study under well-defined conditions which better simulate the situation with clinical ultrasound.

Acknowledgments-The authors gratefully acknowledge the partial support of this study by grants from the National Institutes of Health, GM28253 and GM08209. The authors are also indebted to Dr Marvin Ziskin for helpful comments and suggestions at the beginning of this project. The authors are also grateful to Dr David Boraker and $\mathrm{Dr}$ George Webb for the loan of surgical instruments and for supplying some of the micropipettes used for this study, respectively. The authors also thank Kay Carnes of the Bioacoustics Research Laboratory of the University of Illinois for processing some of the tissue samples for microscopic examination and taking most of the pictures used for this manuscript. One of the authors (LAF) is very much indebted to the other two authors for their hospitality, the use of their facilities, and their generous provision of time and effort which made this study possible.

\section{REFERENCES}

Brown, C. H., Leverett L. B., Lewis C. W., Alfrey C. P. and Hellums J. C. (1975) Morphological, biochemical, and functional changes in human platelets subjected to shear stress. J. Lab. Clin. Med. 86, 462-471.

Chan S. K. and Frizzell L. A. (1977) Ultrasonic thresholds for structural changes in the mammalian liver. In Ultrasonics Symp. Proc., IEEE Cat. \#7CH1264-1SU, 153-156.

Dunn F. and Fry F. J. (1971) Ultrasonic threshold dosages for the mammalian central nervous system. IEEE Trans. Biomed. Engng. BME-18, 253-256.

Frizzell L. A., Lee C. S., Aschenbach P. D., Borrelli M. J., Morimoto R. S. and Dunn F. (1983) Involvement of ultrasonically induced cavitation in the production of hind limb paralysis of the mouse neonate. J. Acoust. Soc. Am. 74, 1062-1065.

Fry F. J., Kossoff G., Eggleton R. C. and Dunn F. (1970) Threshold ultrasonic dosages for structural changes in the mammalian brain J. Acoust. Soc. Am. 48, 1413-1417.
Gross D. R., Miller D. L. and Williams A. R. (1985) A search for ultrasonic cavitation within the canine cardiovascular system. Ultrasound in Med. \& Biol. 11, 85-97.

Lehmann J. F. and Herrick J. F. (1953) Biologic reactions to cavitation, a consideration for ultrasonic therapy. Arch. Phys. Med. Rehab. 34, 86-98.

Martin C. J., Pratt B. M. and Watmough D. J. (1983) Observations of ultrasound-induced effects in the fish Xiphophorous Maculatus. Ultrasound in Med. \& Biol. 9, 177-183.

Miller D. L. (1976) Instrument for microscopical observation of the biophysical effects of ultrasound. J. Acoust. Soc. Am. 60, 12031212.

Miller D. L., Nyborg W. L. and Whitcomb C. C. (1979) Platelet aggregation induced by ultrasound under specialized conditions in vitro. Science 205, 505-507.

National Council on Radiation Protection and Measurements (1983) Biological Effects of Ultrasound: Mechanisms and Clinical Implications. NCRP Report No. 74, Bethesda, MD.

Nyborg W. L. (1958) Acoustic streaming near a boundary. J. Acoust. Soc. Am. 30, 329-339.

Nyborg W. L. (1965) Acoustic streaming. In Physical Acoustics (Edited by W. P. Mason), Vol. IIB, Chap. 11, pp. 265-331. Academic Press, New York.

Stewart H. F. and Stratmeyer M. E. (1982) (eds) An Overview of Ultrasound: Theory, Measurement, Medical Applications, and Biological Effects. HHS Publication FDA 82-8190, Rockville, MD.

ter Haar G. R. and Daniels S. (1981) Evidence for ultrasonically induced cavitation in vivo. Phys. Med. Biol. 26, 1145-1149.

ter Haar G. R., Daniels S., Eastaugh F. C. and Hill C. R. (1982) Ultrasonically induced cavitation in vivo. Br. J. Cancer 45, Suppl. V, 151-155.

Williams A. R., Hughes D. E. and Nyborg W. L. (1970) Haemolysis near a transversely oscillating wire. Science 169, 871-873.

Williams A. R. (1977) Intravascular mural thrombi produced by acoustic microstreaming. Ultrasound in Med. \& Biol. 3, 191203.

World Health Organization (1982) Environmental Health Criteria 22: Ultrasound. World Health Organization, Geneva. 